Research Paper

\title{
7,8-Dihydroxyflavone alleviates apoptosis and inflammation induced by retinal ischemia-reperfusion injury via activating TrkB/Akt/NF-kB signaling pathway
}

\author{
Aihua $\mathrm{Yu}^{1,2 \#}$, Shun Wang ${ }^{1,2 \#}$, Yiqiao Xing ${ }^{\circledR}$, Mengyao Han², Kun Shao² \\ 1. Eye Center, Renmin Hospital of Wuhan University, Wuhan University, Wuhan 430060, Hubei Province, China \\ 2. Department of Ophthalmology, Zhongnan Hospital of Wuhan University, Wuhan University, Wuhan 430071, Hubei Province, China \\ \# These authors contribute equally to this work \\ $\square$ Corresponding author: Dr. Yiqiao Xing, Department of Ophthalmology, Renmin Hospital of Wuhan University, Wuhan 430060, Hubei Province, China; \\ email: Yiqiao_xing57@whu.edu.cn
}

(c) The author(s). This is an open access article distributed under the terms of the Creative Commons Attribution License (https://creativecommons.org/licenses/by/4.0/). See http://ivyspring.com/terms for full terms and conditions.

Received: 2021.08.05; Accepted: 2021.10.26; Published: 2022.01.01

\begin{abstract}
Retinal ischemia-reperfusion injury (RIRI) is of common occurrence in retinal and optic nerve diseases. The BDNF/TrkB signaling pathway has been examined to be neuroprotective in RIRI. In this study, we investigated the role of a potent selective TrkB agonist 7,8-dihydroxyfavone (DHF) in rat retinas with RIRI. Our results showed that RIRI inhibited the conversion of BDNF precursor (proBDNF) to mature BDNF (mBDNF) and increased the level of neuronal cell apoptosis. Compared with RIRI, DHF+RIRI reduced proBDNF level and at the same time increased $m B D N F$ level. Moreover, DHF administration effectively activated TrkB signaling and and downstream Akt and Erk signaling pathways which increased nerve cell survival. The combined effects of mBDNF/proBDNF increase and TrkB signaling activation lead to reduction of apoptosis level and protection of retinas with RIRI. Moreover, it was also found that astrocytes labeled by GFAP were activated in RIRI and NF-kB mediated the increased expressions of inflammatory factors and these effects were partially reversed by DHF administration. Besides, we also used RNA sequencing to analyze the differently expressed genes (DEGs) and their enriched (Kyoto Encyclopedia of Genes and Genomes) KEGG pathways between Sham, RIRI, and DHF+RIRI. It was found that 1543 DEGs were differently expressed in RIRI and 619 DEGs were reversed in DHF+RIRI. The reversed DEGs were typically enriched in PI3K-Akt signaling pathway, Jak-STAT signaling pathway, NF-kB signaling pathway, and Apoptosis. To sum up, the DHF administration alleviated apoptosis and inflammation induced by RIRI via activating TrkB signaling pathway and may serve as a promising drug candidate for RIRI related ophthalmopathy.
\end{abstract}

Key words: Retinal ischemia-reperfusion injury; 7,8-dihydroxyfavone; BDNF; TrkB; Apoptosis.

\section{Introduction}

Retinal ischemia-reperfusion injury (RIRI) is the recovery of perfusion after acute ischemia of the retina, causing irreversible structural and metabolic changes in the retinal tissue [1]. It is easy to damage the retinal nerve tissue and cause varying degrees of visual function impairment. Acute retinal ischemia is clinically common in acute high intraocular pressure, retinal vascular embolism disease and intraocular surgery [2].

At present, the mechanism of RIRI mainly includes various theories such as apoptosis, increase of free radicals and inflammatory factors, and decrease of neurotrophic factors [3]. Brain derived neurotrophic factor (BDNF) is a protein synthesized by nerve cells and is mainly distributed in the central nervous system and peripheral nervous system. The main function of BDNF is to affect the development, growth and differentiation of retinal neuron cells during the embryonic period, and to have a certain impact on the survival of differentiated ganglion cells 
[4]. Current studies have shown that when culturing retinal nerve cells in vitro, BDNF can increase the number of nuclei and the thickness of the plexiform layer, and at the same time promote the formation and maturation of synapses by regulating the bifurcation of dendrites and axons [5,6]. In the retinal ischemia-reperfusion model, BDNF plays a very important role in the functional recovery of experience-dependent retinal cells [7]. In animal models of retinal ischemia-reperfusion, early injection of BDNF into the vitreous cavity can reduce the apoptosis of retinal nerve cells. The Schwann cells transfected with BDNF gene were injected into the vitreous cavity to elevate BDNF expression in the retina, and it was found that BDNF has the effect of nourishing retinal nerve cells [8]. The post-translation processing of BDNF happened in the nervous system, that is, precursor of BDNF (proBDNF) is converted into mature BDNF (mBDNF) by shearing action of calcium-dependent protease Furin [9]. ProBNDF and mBDNF have completely different physiological functions. ProBDNF can induce the apoptosis of nerve cells by activating p75NTR signaling pathway, while mBDNF can promote the formation of axons and dendrites of nerve cells and delay the apoptosis of nerve cells through activating TrkB signaling pathway $[10,11]$.

Due to the protective roles of BDNF in nerve cells, any changes involved in the production, maturation and signal transduction pathways of BDNF will cause damage to the optic nerve. 7,8-Dihydroxyflavone (DHF) is a potent mimetic material of BDNF and has been exhibited neuroprotective properties in several retinal injury models [12-14]. However, there is still lacking evidences to support its neuroprotective roles in RIRI. In this study, RIRI models of rats were established and the pathological changes were observed in the retina tissue. Moreover, DHF was used to treat RIRI rats and the responses of DHF administration in the injured retina were detected by RNA-seq analysis. We want to investigate whether DHF can exhibit neuroprotective functions in RIRI and the related mechanism of its action.

\section{Materials and methods}

\section{Animals}

Healthy male Sprague-Dawley (SD rats), 7-week-old and weighted 200-220 g, were supplied by the Animal Experimental Center of Zhongnan Hospital, Wuhan University, and housed in SPF grade animal feeding rooms (temperature $20-25^{\circ} \mathrm{C}$, humidity $40 \%$ ) under a $12 \mathrm{~h}-12 \mathrm{~h}$ of light to dark cycle condition. All the animal experiments were ratified by the
Animal Experiment Center and Ethics Committee of Zhongnan Hospital of Wuhan University and also followed the National Institutes of Health Guide for the Care and Use of Laboratory Animals. There was no obvious abnormality for all animals in ocular examination and general condition. General condition requirements: hair with color bright, limbs without obvious fracture or scar and disease signs. Eye examination: the eyelids of both eyes are complete, the cornea was bright, the anterior chamber was visible, the pupil was round without obvious abnormal pupil shape, and the iris was white. All rats were routinely fed for 7 days before the experiment to adapt to the environment.

\section{Rat model of retinal ischemia-reperfusion injury}

Before the experiment, the animals were weighted and narcotized by abdominal cavity injection with $10 \%$ (w : v) chloral hydrate $(3 \mathrm{~mL} / \mathrm{kg})$, then 3 drops of levofloxacin were dropped on to the eyes, and the right eye was anesthetized to dilate the pupils with tropicamide and prometvacaine hydrochloride. Ofloxacin ophthalmic gel was used to cover the cornea of the rats after anterior chamber puncture in the left eye, which was used as the Sham group. A 30-gauge sterile needle connected to a sterile plastic infusion tube was pricked into the anterior chamber of right eye horizontally, and the position of the needle was fixed. Then a normal saline bag attached to the other end of the infusion tube was raised to a height of $150 \mathrm{~cm}$, which can make the intraocular pressure increase to about $110 \mathrm{mmHg}$ in the eyes. The needle was removed gradually until the ischemic condition was kept for $60 \mathrm{~min}$. The conjunctival congestion could be seen after the needle was pulled out, and the retina of the rats changed from white to orange, indicating that the retinal blood vessels had resumed perfusion. Propivacaine hydrochloride and levofloxacin were continuously dropped on to the eyes during the maintenance of high pressure perfusion. After needle removal, ofloxacin gel was applied to cover the conjunctiva of the rat to prevent infection. Animals were killed respectively at 0 hours, 6 hours, 24 hours, 72 hours, and 144 hours after RIRI, retinas or eyeballs were collected for the following analysis in Figure 1.

\section{Drug Administration}

For the Sham group, ischemia-reperfusion was not done and the solvent (10\% DMSO and $90 \%$ corn oil) was applied by intraperitoneal injection. In the RIRI group, ischemia-reperfusion was induced and then the solvent was also injected by intraperitoneal injection. For 7,8-Dihydroxyflavone (DHF)+RIRI 
group, DHF (dissolved in 10\% DMSO and $90 \%$ corn oil), $10 \mathrm{mg} / \mathrm{kg}$ (MCE, USA), was injected intraperitoneally after completion of the ischemic condition. Six days (144 hours) later, retinas or eyeballs were collected for the following analysis in Figure 2-6.

\section{Hematoxylin and eosin staining and quantification of the retinal thickness}

After the RIRI model was constructed, eyes were enucleated from anesthetized rats. The conjunctiva, the muscle and connective tissue outside the eye were removed. After removing extraocular tissues, the samples were fixed in $4 \%$ paraformaldehyde for 2 hours, then fixed in $4 \%$ neutral paraformaldehyde, embedded in paraffin and sectioned. Slides were stained by hematoxylin solution for 90 seconds followed by another water wash for $15 \mathrm{~min}$. Then, slides were stained by eosin solution for 30 seconds, and dehydrated by ethanol. Mounting medium was applied once slides were dried and the slides were cover-slipped and photographed under a light microscope. Five points of retinal or inner nuclear layer (INL) thickness were measured in a section at least by Digimizer (https://www.digimizer.com/ index.php), and evaluated the averages of them as the value of an eye. All the data were calculated and showed by GraphPad Prism 8 .

\section{Transmission electron microscope for mitochondria}

Fresh retina tissues with total volume less than 1 $\mathrm{cm}^{3}$ were fixed for 2-4 h in 3\% glutaraldehyde under 4 ${ }^{\circ} \mathrm{C}$, then washed 3 times with $0.1 \mathrm{M}$ PBS buffer ( $\mathrm{pH}=7.4$ ) and 15 minutes for each. $1 \%$ osmic acid was used for fixing until 2 hours under room temperature, after that washed 3 times with $0.1 \mathrm{M}$ PBS. The samples will be dehydrated with the gradient alcohol, such as 50\%-70\%-80\%-90\%-95\%-100\%-100\%, and 15 minutes for each. Then the samples will be incubated with the solution (acetone : spon812 = $1: 1$ ) over night, and polymerized for 48 hours under $60{ }^{\circ} \mathrm{C}$. The samples for ultrathin section $(60-70 \mathrm{~nm})$ were stained with uranyl acetate and lead citrate, and then were observed and photographed under a transmission electronic microscope (JEOL JEM-1400plus, Japan).

\section{Western blot assay}

After isolation of retina, the tissue samples were grinded into fine powder in liquid nitrogen with a mortar and pestle. The cell lysis buffer for Western and IP (Beyotime Biotech, China), mixed with protease and phosphatase inhibitor cocktail (50X, P1050, Beyotime Biotech, China) for mammalian cell and tissue extracts, was applied to the powder for cell lysis (one hour on the ice). Protein concentrations were measured using NanoPhotometer N60. $50 \mu \mathrm{g}$ protein sample per lane was separated on $10 \%$ SDS-PAGE and then was transferred onto a PVDF membranes (Millipore, USA). The membranes were blocked in 5\% skim milk in Tris-buffered saline with $0.5 \%$ Tween 20 (TBST) for 1-2 hours at room temperature on a shaking table. The membrane was incubated overnight at $4{ }^{\circ} \mathrm{C}$ with primary the antibodies, including mBDNF (ABclone, 1 : 1000, China), proBDNF (Santa, 1 : 500, USA), TrkB (CST, 1 : 1000), pTrkB (CST, 1: 1000, USA), Akt (CST, 1: 1000), pAkt (CST, 1: 1000), Erk1/2 (CST, 1: 1000), pErk1/2 (CST, 1: 1000) Caspase3 (ABclone, 1 : 1000), Bax (ABclone, 1 : 1000), Bcl-2 (CST, 1: 1000), GFAP (ABclone, 1 : 1000), NF-kB (ABclone, 1 : 1000), Phospho-NF-kB (ABclone, 1 : 1000) and GAPDH (ABclone, $1: 1000)$. On the next day, after washing with TBST by 3 times and $5 \mathrm{~min}$ for each, membranes were incubated with horseradish peroxidase conjugated secondary antibody (1 : 5000, Beyotime Biotech, China) on the shaking table for 2 hours at room temperature. The membranes will be washed with TBST, and after that, protein bands were detected by using ECL reagent (Vazyme Biotech Co.,Ltd, China) and obtained images after exposure on Bio-Rad ChemiDoc XRS ${ }^{+}$. With the help of Image $\mathrm{Lab}$, an image analysis software, the intensity of the protein band was semiquantitatively measured. GAPDH was used for internal reference.

\section{Immunofluorescence assay}

An In situ Cell Apoptosis Detection Kit (Tunel assay, Boster,Wuhan, China) was used to monitor the apoptotic positive cells on the paraffin-embedded retina sections. We chose five different areas in each retinal section to count the Tunel-positive cells. The positive cell numbers per unit length of retinal surface in the 5 areas were reported. Three samples per group were used in this assay.

Paraffin section of retina was incubated under 65 ${ }^{\circ} \mathrm{C}$ for $2 \mathrm{~h}$, and then washed with PBS (0.01M) by 3 times after dewaxing and $5 \mathrm{~min}$ for each. Antigen retrieval was finished with EDTA repair liquid and then washed with PBS by 3 times. 3\% peroxide was used to incubate for the samples for $10 \mathrm{~min}$ in dark. The sections were incubated overnight at $4{ }^{\circ} \mathrm{C}$ with anti-GFAP (ABclone, $1: 200$ ), and after washing with PBS, they were incubated with secondary antibody for $1 \mathrm{~h}$. Finally, the sections were incubated with DAPI before covered with coverslip and observed under fluorescence microscope. The average flourescence intensity was calculated by Image J (v1.46r). 


\section{RNA-seq analysis and qRT-PCR confirmation}

Total RNA was extracted using TRIzol reagent (Invitrogen, Carlsbad, CA, USA) following the manufacturer's procedure. $5 \mu \mathrm{g}$ total RNA was used to purify the poly(A) RNA by using Dynabeads Oligo (dT)25-61005 (Thermo Fisher, CA, USA). Then the poly(A) RNA was used for construction of cDNA library. At last, $2 \times 150$ bp paired-end sequencing (PE150) was performed on an Illumina Novaseq ${ }^{\mathrm{TM}}$ 6000 (LC-Bio Technology CO., Ltd., China) following the vendor's recommended protocol.

Cutadapt software was used to get rid of the reads that contained adaptor contamination. HISAT2 software was used to map the reads to the rat genome (ftp://ftp.ensembl.org/pub/release-101/fasta/rattus _norvegicus/dna/). Then, StringTie was used to assemble the mapped reads came from each sample with default parameters. And, all transcriptomes were merged to reconstruct a comprehensive transcriptome using gffcompare software. After that, StringTie and ballgown were used to estimate the expression levels and perform expression level for mRNAs by calculating FPKM. The differentially expressed mRNAs were screened with $\mid \log 2$ (fold change) $\mid>0.5$ and $p$ value $<0.01$ by $R$ package edge $R$, and then were used for GO enrichment and KEGG enrichment analysis. The heatmap, venny analysis, GO and KEGG enrichment analysis were performed by OmicStudio (https://www.omicstudio.cn/).

qRT-PCR was performed according to a previous study [15] and primers used in this study were showed in supplemental file Table S3.

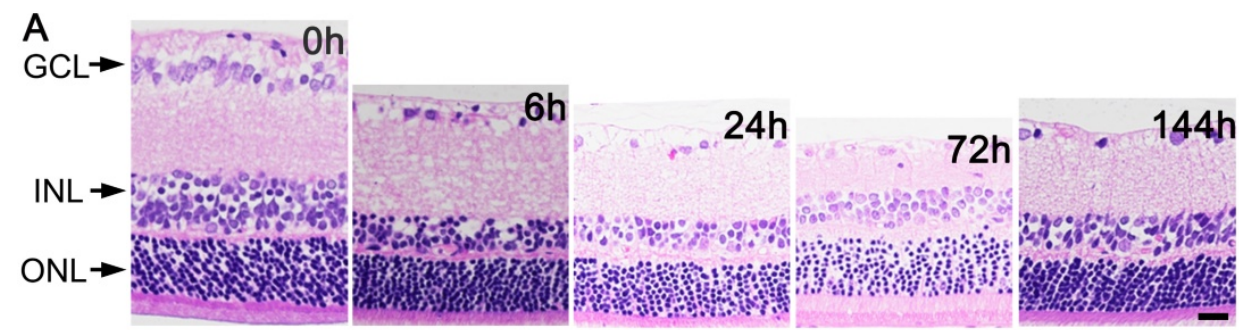

B
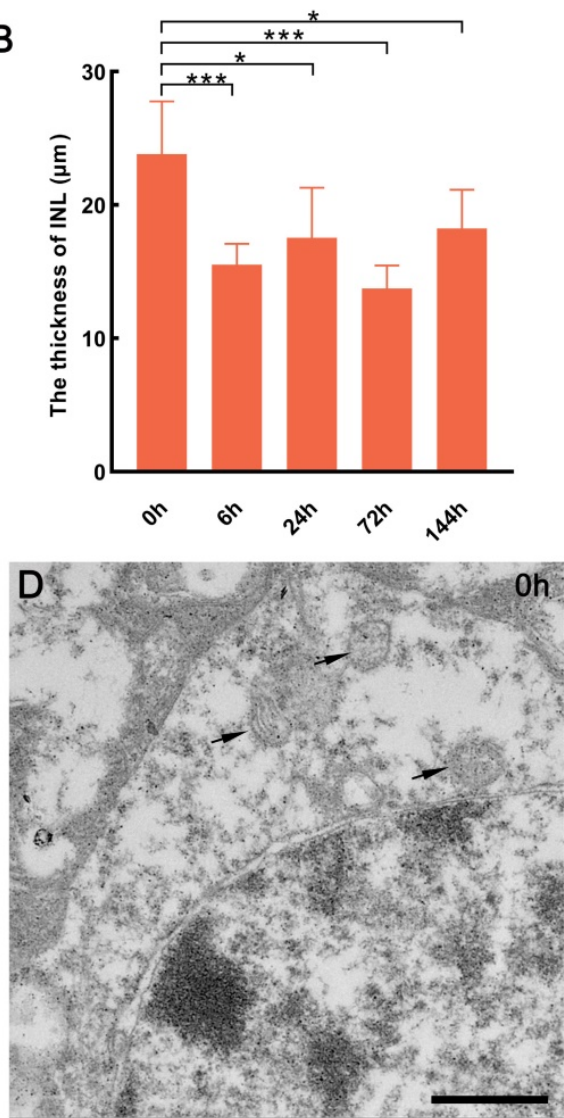

C
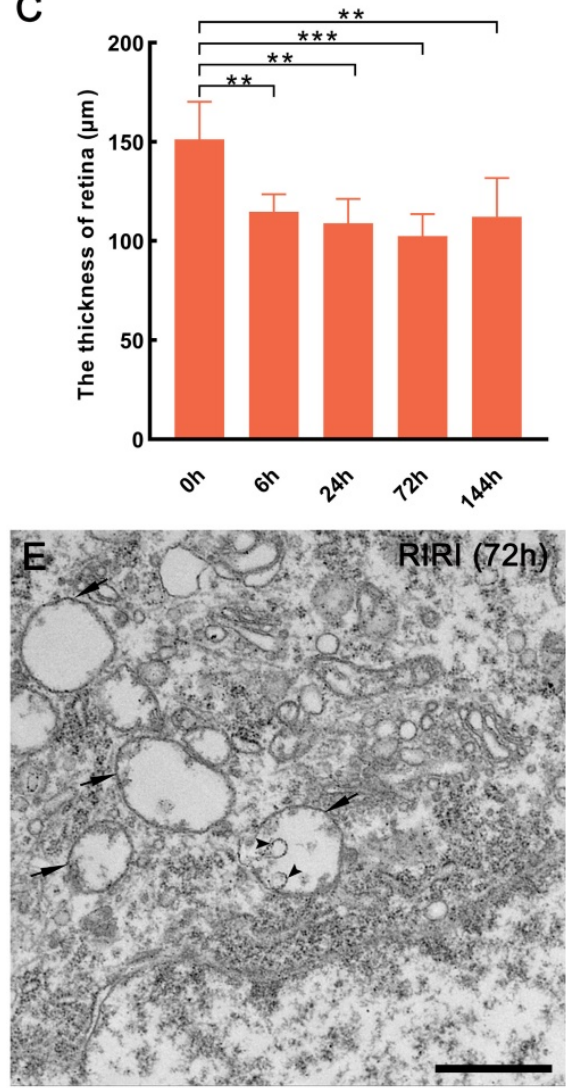

Figure 1. Histologic and mitochondrial changes of the retina induced by RIRI in rats. (A) Hematoxylin and eosin staining of retina for $0 \mathrm{~h}$ and different reperfusion time group. $(B$ and $C$ ) The statistical data on thickness of inner nuclear layer (INL), and the whole retina. Scale bar: $20 \mu \mathrm{m}(A)$. (D and E) Mitochondrial structure observation of $0 \mathrm{~h}$ and RIRI after $72 \mathrm{~h}$. Arrows show the mitochondria and the arrow heads show the shedding inner membrane in mitochondria. GCL: ganglion cell layer. ONL: outer nuclear layer. $* * * \mathrm{p}<$ 0.001 , **P $<0.01$, $* \mathrm{P}<0.05$, ANOVA test was used for comparing the values between contol $(0 \mathrm{~h})$ and RIRI series $(6 \mathrm{~h}, 24 \mathrm{~h}, 72 \mathrm{~h}$, and $144 \mathrm{~h})(\mathrm{n}=5 \mathrm{determinations}$ for each). Scale bars: $1 \mu \mathrm{m}$ (D and $E)$. 


\section{Results}

\section{Retina changed apparently after retinal ischemic-reperfusion injury in rat}

To gain insight into the characterization of retinal ischemic-reperfusion injury (RIRI), we established an in vivo rat model under different reperfusion time and observed the tissue structure integrity by H\&E staining. The result showed that the cell morphology and volume of ganglion cell layer (GCL) had apparent changes with different reperfusion time (Figure 1A). At the same time, the inner nuclear layer (INL) was thinner in the RIRI group compared with $0 \mathrm{~h}$ group, the cells were arranged sparsely, especially at $6 \mathrm{~h}$ after ischemic-reperfusion injury (Figure $1 \mathrm{~A}$ and $\mathrm{B}$ ). Moreover, the cells of outer nuclear layer (ONL) more sparsely arranged as well after 24 and 72 hours ischemic-reperfusion (Figure 1A). In addition, we also found that the whole retina was thinner after RIRI occurred than before (Figure $1 \mathrm{~A}$ and $\mathrm{C}$ ). As a result, RIRI can obviously change the tissue structure of retina in rats, and it's meaningful to make clear about how this process was regulated.

The structure of mitochondria was also observed in our study. The result showed that in $0 \mathrm{~h}$ group, the mitochondria maintained intact structure including abundant mitochondrial matrix and cristae (Figure 1D), however, we found many vacuolar mitochondria with very few matrix in the RIRI group (72 h) (Figure $1 \mathrm{E})$. Sometimes, in the abnormal mitochondria, some circled structure, formed by inner membrane shedding from mitochondrial outer membrane, will be observed (Figure 1E). As a result, RIRI can disrupt mitochondrial membrane structure in rat retina.

\section{7, 8-Dihydroxyflavone protected the retina after RIRI}

In order to confirm the role of BDNF in RIRI response, a selective receptor agonist for TrkB, 7, 8-Dihydroxyflavone (DHF), was used for RIRI. The results of HE staining (Figure 2A) and statistical analysis (Figure 2B) showed that the thickness of INL and the whole retina were thinner in RIRI group than those in Sham group. However, in DHF+RIRI group, the thickness of INL and retina were almost the same as the Sham group (Figure 2A and B). We also did TUNEL assay, and found that the increased TUNEL signal in RIRI was reduced after DHF treatment (Figure 2C and D).

Glial fibrillary acidic protein (GFAP) is a marker for activated astrocytes [16]. Activated glial cells expressing increased GFAP levels were detected in glaucoma animal models [17] and human glaucomatous donor eyes [18]. In the RIRI group, the GFAP signals were increased compared with Sham. But after DHF treatment, GFAP expression reduced to approximately the same as Sham (Figure $3 \mathrm{~A}$ ). The results of qRT-PCR (Figure 3B) and western blot (Figure $3 \mathrm{C}$ and $\mathrm{D}$ ) also confirmed these findings. NF-kB and phosphorylated NF-kB (pNF-kB) were also tested by western blot in our research. And the results showed that pNF-kB/NF-kB was significantly increased in RIRI and almost recovered by DHF (Figure 3E and F). At mRNA level, we also found that some inflammatory factors, such as IL-1 $\beta$, IL-6, TNF- $\alpha$, and IFN- $\gamma$ were all induced by RIRI and restored after DHF treatment (Figure 3G).

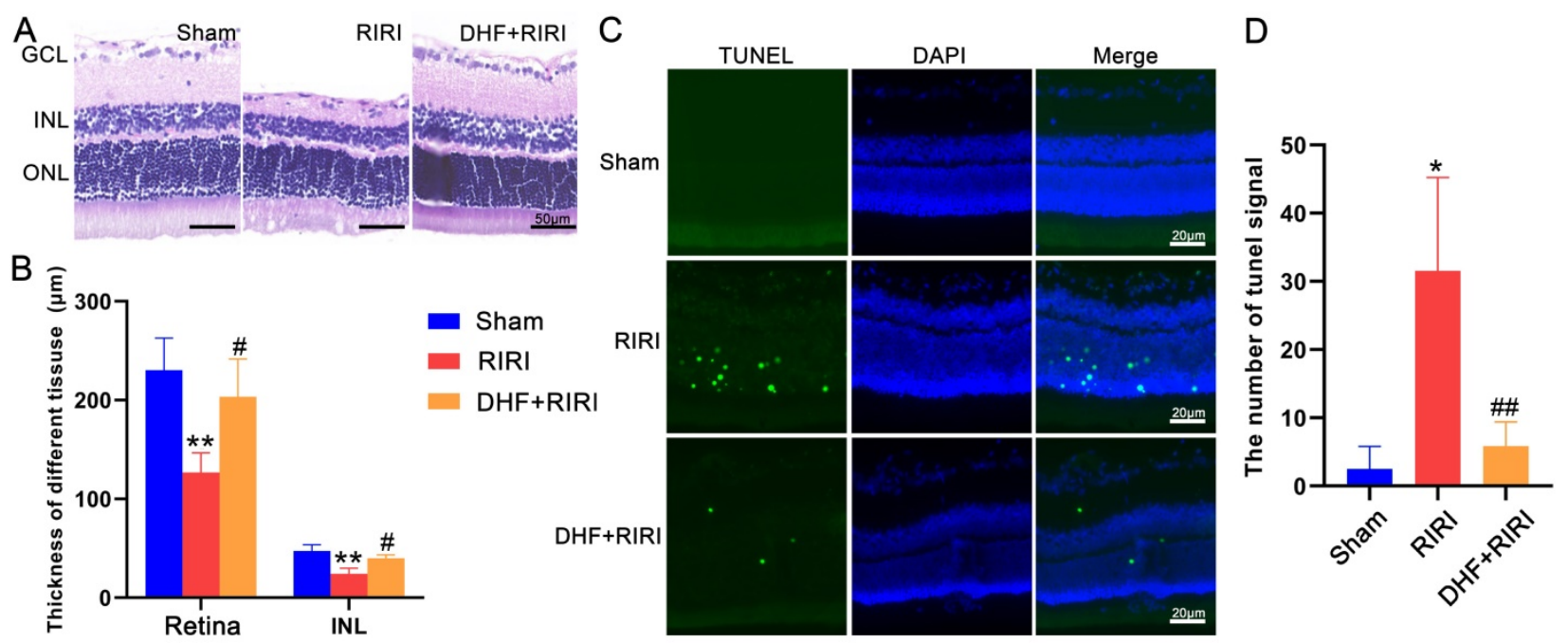

Figure 2. The effect of 7, 8-Dihydroxyflavone on RIRI in rat. (A) Hematoxylin and eosin staining of retina for Sham, RIRI, and DHF+RIRI groups. (B) The statistic of thickness of inner nuclear layer (INL) and the whole retina in different groups. (C and D) TUNEL test and statistic of TUNEL signal in Sham, RIRI, and DHF+RIRI samples. GCL: ganglion cell layer. ONL: outer nuclear layer. ${ }^{* * P}<0.01$, ${ }^{*} \mathrm{P}<0.05$ for RIRI compared with Sham, \#\#P $<0.01$, \#P $<0.05$ for DHF+RIRI compared with RIRI, by T-test $(\mathrm{n}=5$ determinations for each). Scale bar: $50 \mu \mathrm{m}(\mathrm{A}), 20 \mu \mathrm{m}(\mathrm{C})$. 


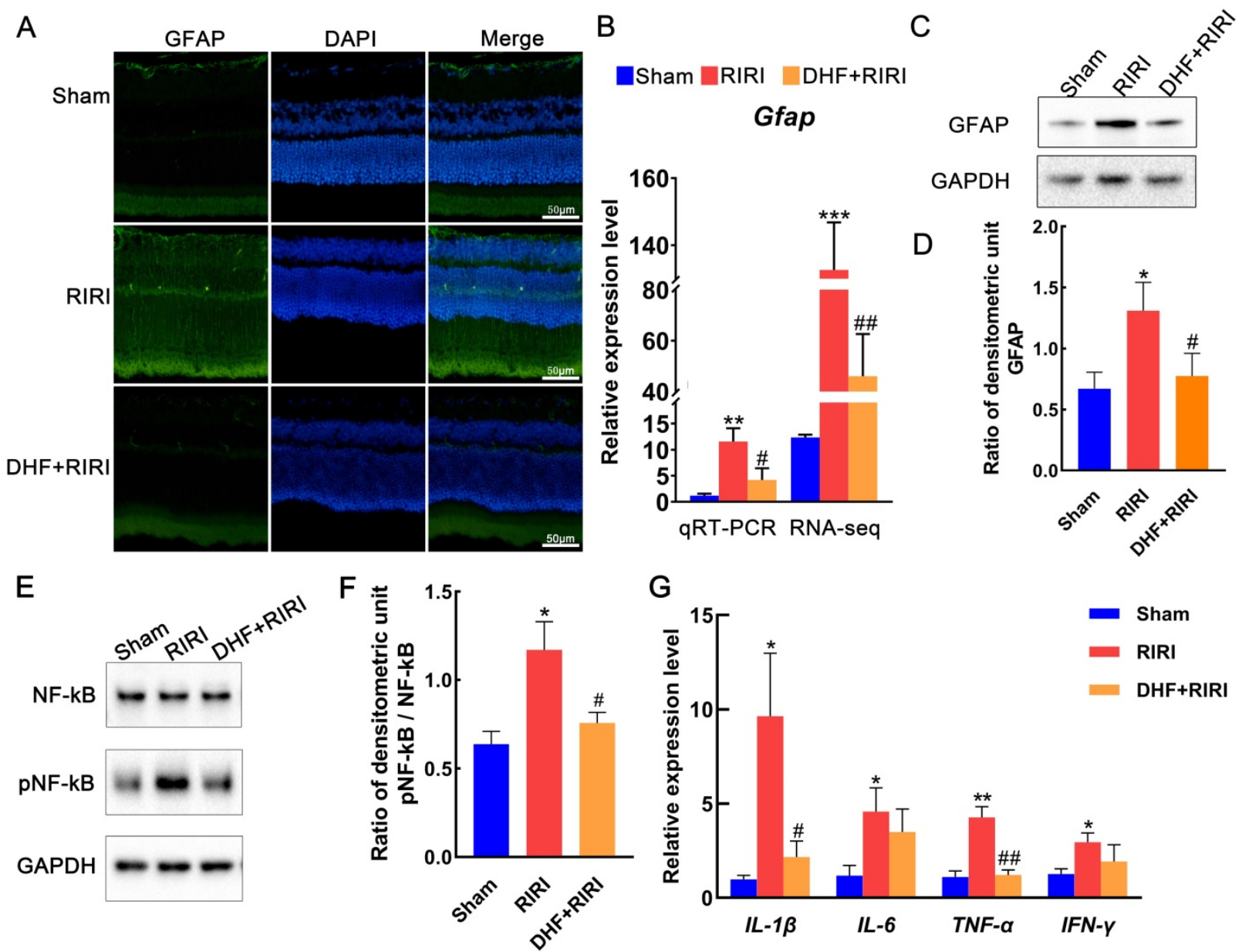

Figure 3. The alterations of GFAP expression in RIRI and DHF+RIRI. (A) Immunofluorescence analysis of anti-GFAP in Sham, RIRI and DHF+RIRI. (B) $q R T-P C R$ analysis and RNA-seq data for GFAP in different conditions. (C and D) Western blot analysis and statistics of GFAP in different conditions. (E and F) Western blot analysis and statistics on pNF-kB and NF-kB. (G) qRT-PCR analyses for IL-I $\beta, I L-6, T N F-\alpha$, and IFN- $y$. ***P $<0.001$, **P $<0.01$, *P $<0.05$ for RIRI compared with Sham, \#P $<0.01$, \#P $<0.05$ for DHF+RIRI compared with RIRI, by T-test $(\mathrm{n}=3$ determinations for each). Scale bar: $50 \mu \mathrm{m}$.

\section{Expression profile analysis of rat retinas in RIRI and DHF+RIRI}

To explore the molecular mechanism in rat RIRI, RNA-seq analysis was carried out based on Sham, RIRI and DHF+RIRI samples. Through whole transcriptome profiling, we identified a cascade of genes and pathways potentially involved in DHF effects. 1234 deferentially expressed genes (DEGs) were up-regulated and 309 DEGs were down-regulated in RIRI compared with Sham (Figure 4A and Table S1), and 103 DEGs were up-regulated and 664 genes were down-regulated due to the effect of DHF (Figure 4A and Table S1) ( $|\log 2 \mathrm{FC}|>0.5$ and $p<0.01)$. Among the 1543 DEGs in RIRI, there were 619 DEGs were reversed by DHF administration, including 575 up-regulated genes and 44 down-regulated genes (Figure 4B and Table S1). The heat map and clustering analysis based on the expression of 619 genes was also performed (Figure $4 \mathrm{C})$. These results suggested that the DHF may reverse the expression of these genes and these genes may play essential role in RIRI response.

To further understand the function of these genes, Gene Ontology (GO) and Kyoto Encyclopedia of Genes and Genomes (KEGG) analysis were done. The 619 DEGs reversed by DHF were enriched in inflammatory response, immune system process, immune response, phagocytosis, positive regulation of angiogenesis, positive regulation of ERK1 and ERK2 cascade, lysosome and some endosome related GO term (Figure 4D and Table S2). These genes were also enriched in phagosome, cell adhesion molecules (CAMs), lysosome, apoptosis, necroptosis, PI3K-Akt signaling pathway, Jak-STAT signaling pathway, and some immune-related pathways (Figure 4E and Table S2). These results suggested that DHF may alleviate RIRI by affected the inflammatory response, immune response, and the BDNF/TrkB signaling pathway.

To make sure about the RNA-seq result, the expression levels of a set of genes, selected from the reversed DEGs, were assayed by quantitative real-time PCR (qRT-PCR). It was found that 9 genes (Casp1, Bcl2a1, Ccl20, Il17ra, Pycard, Rac2, Tgfb1, Myc, 
and Lgals3) were significantly up-regulated in RIRI, and 8 of them were significantly down-regulated in DHF+RIRI and the expression level of 7 DEGs was almost recovered with the same as Sham (Figure 5). Moreover, there were 2 genes (Nefm and Pak1) down-regulated in RIRI and reversed by DHF (Figure 5). These results proved the RNA-seq analysis was credible.

\section{BDNF/TrkB signaling pathway is activated in RIRI after DHF administration}

To make clear about the role of BDNF-TrkB signaling in RIRI regulation, the expression levels of BDNF and TrkB in retina after RIRI were firstly tested by western blot. Interestingly, the precursor form of brain-derived neurotrophic factor (proBDNF) was increased after RIRI but reduced after DHF treatment, while the mature BDNF showed reverse expression pattern (Figure 6A and $\mathrm{B}$ ). As a ligand for mature BDNF, the TrkB did not have any expression changes, however, the expression of phosphorylated TrkB (pTrkB) was lower in RIRI than Sham and restored by DHF treatment (Figure 6A and B). The downstream factors, pAkt/Akt and pErk/Erk, were also monitored in different groups in our study. They all inhibited by RIRI and can be restored by DHF (Figure 6A and B). Caspase 3 and Bax increased in RIRI but restored by DHF, however Bcl-2 was inhibited in RIRI and also restored by DHF (Figure 6C and D). These results suggested that DHF activated BDNF/TrkB signaling pathway and reduced the level of apoptosis induced by RIRI.
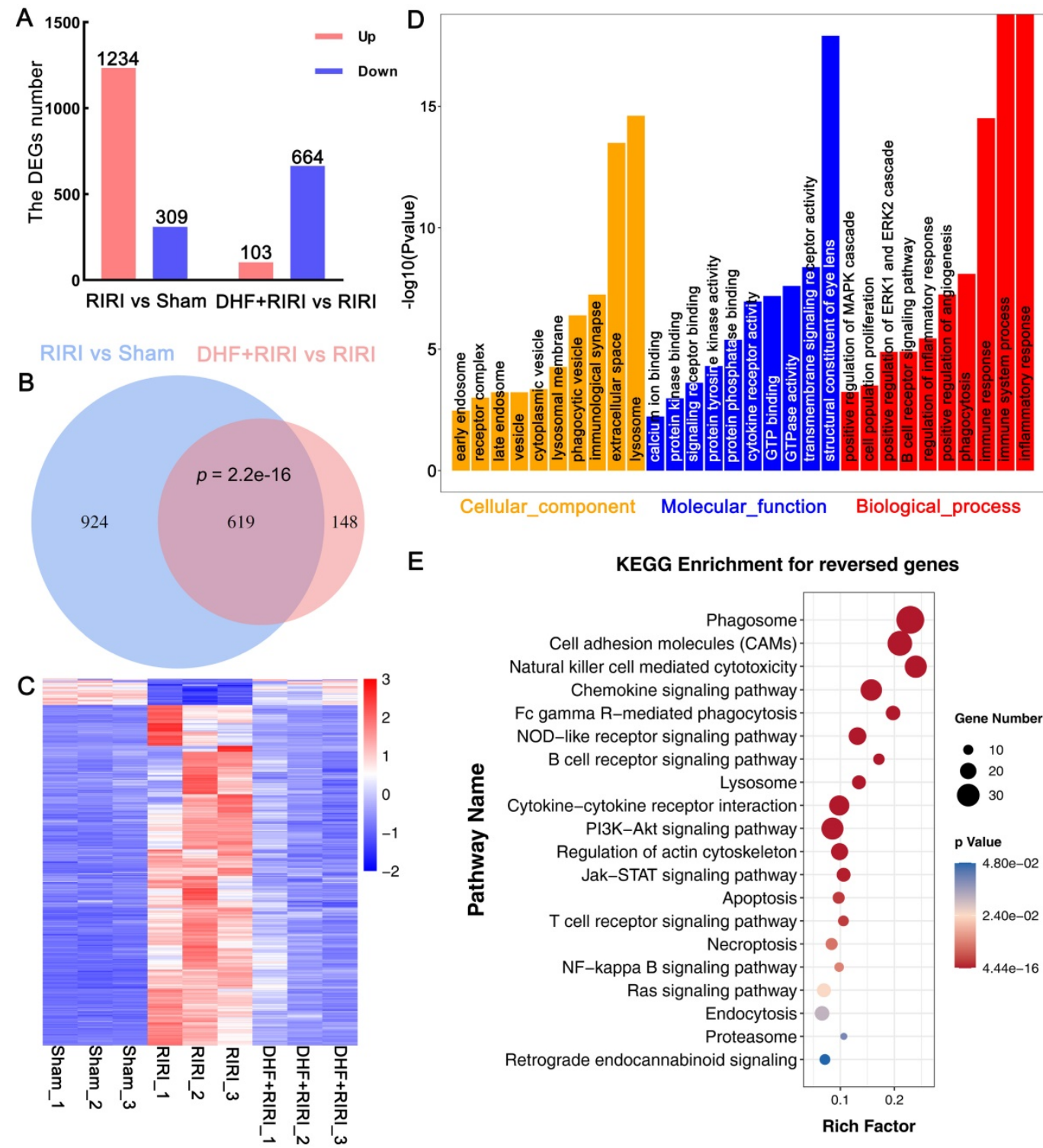

E

KEGG Enrichment for reversed genes

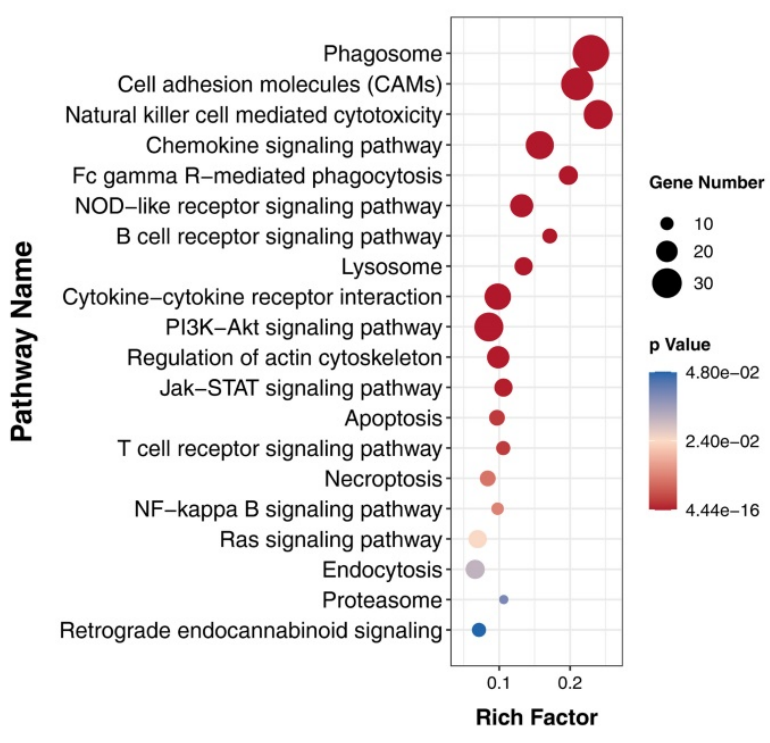

Figure 4. RNA-seq analysis on Sham, RIRI, and DHF+RIRI in rat. (A) Histogram of deferentially expressed genes (DEGs) between different groups. (B) Venny analysis between DEGs in RIRI vs Sham and DEGs in DHF+RIRI vs RIRI. (C) Expression heatmap of 619 reversed genes. (D and E) GO enrichment analysis and KEGG enrichment analysis based on the reversed genes. 


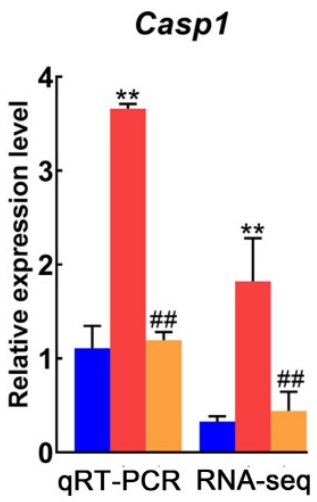

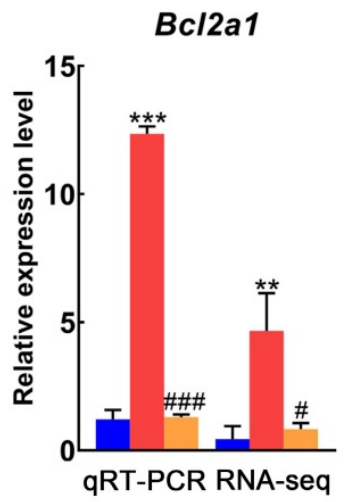

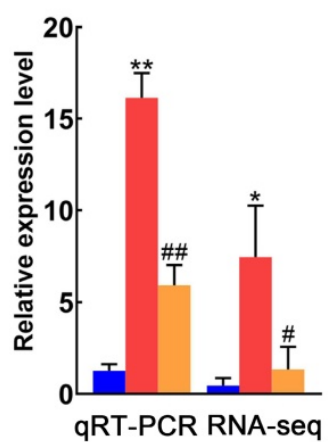

Nefm

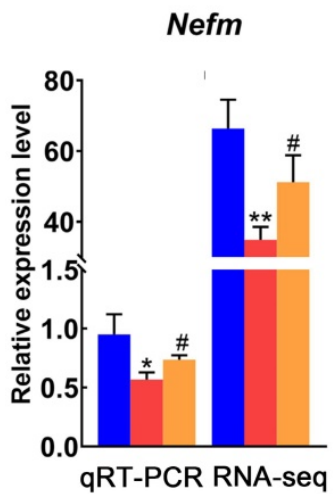

Pak1

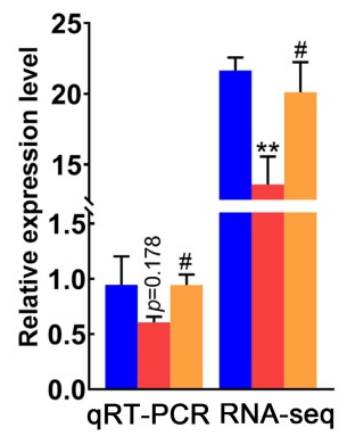

Cc/20

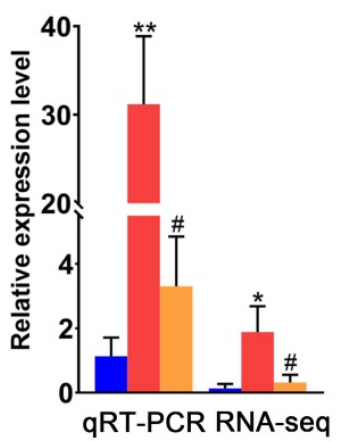

Tgfb1

II17ra

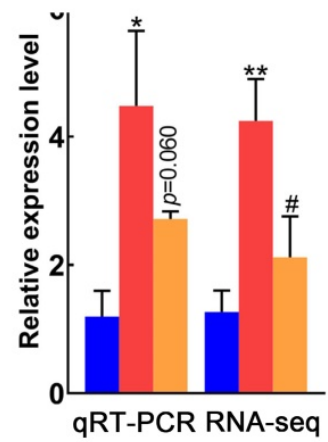

Myc
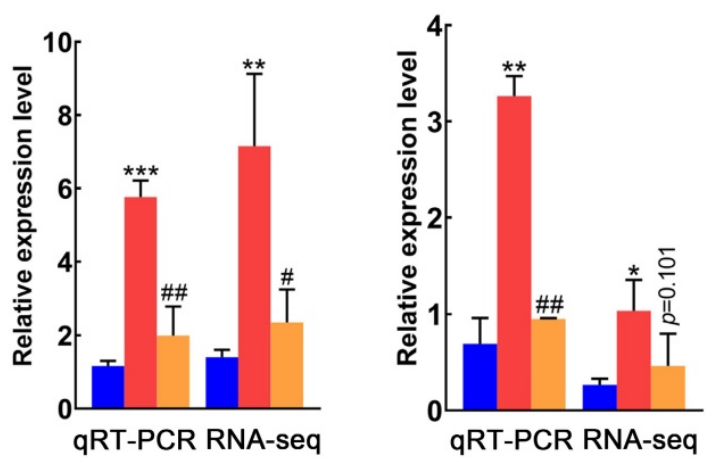

Lgals3

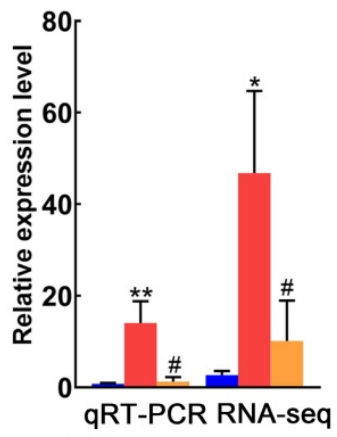

Sham

RIRI

DHF+RIRI

Figure 5. Expression validation of differently expressed genes by $\mathrm{QRT}-\mathrm{PCR}$. ***P $<0.001$, **P $<0.01, * \mathrm{P}<0.05$ for RIRI compared with Sham, \#\#P<0.001, \#P $<0.01$, \#P $<$ 0.05 for DHF+RIRI compared with RIRI, by T-test ( $\mathrm{n}=3$ determinations for each).

\section{Potential relationship between Furin and BDNF}

Furin maybe an important regulator of BDNF (30-33). To validate this, we detected their expression level in retina by immunofluorescence. The results showed that Furin and BDNF colocalized in most areas in retina and both of them were deduced after RIRI (Figure 7). These results suggested that the expressions of Furin and BDNF were positively correlated and Furin might execute the role of processing proBDNF to mBDNF.

\section{Discussion}

Retinal ischemia-reperfusion injury (RIRI) is a common pathological change in ophthalmology and many ophthalmic diseases with high intraocular pressure cause damages to visual function through several mechanisms include inflammatory cascade, free radicals and calcium overload [19]. In this study, we investigate the neuroprotective effects of DHF on retinal and optic neurons in RIRI rat models for the first time. We confirmed that RIRI could increase apoptosis and inflammation level in the retinas of rat. DHF inhibit astrocyte activation, inflammation and apoptosis level in RIRI by activating TrkB/Akt/NF-kB signaling and regulating Bcl-2/Bax apoptosis signaling. 

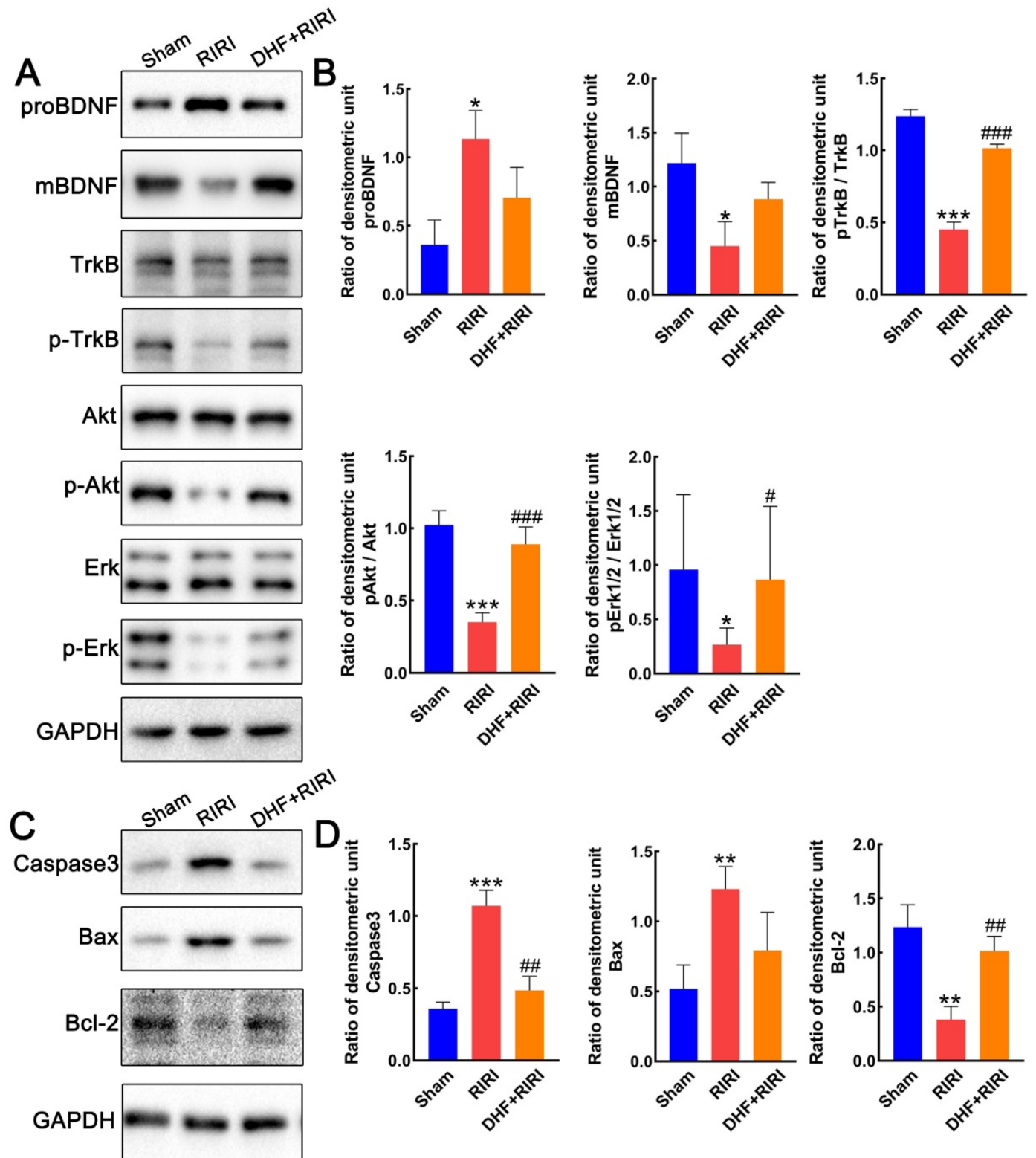

Figure 6. Expression alteration of BDNF/TrkB and apoptosis pathway. (A and B) Western blot analysis and statistics on proBDNF, mBDNF, TrkB, $\mathrm{pTrkB}$, Erk1/2, pErk1/2. (C and D) Western blot analysis and statistics on Caspase3, Bax, and Bcl-2. ${ }^{* * * P}<0.001, * * P<0.01, * P<0.05$ for RIRI compared with Sham, \#\# 0.001 , \#P $<0.01$, \#P $<0.05$ for DHF+RIRI compared with RIRI, by T-test ( $\mathrm{n}=3$ determinations for each).
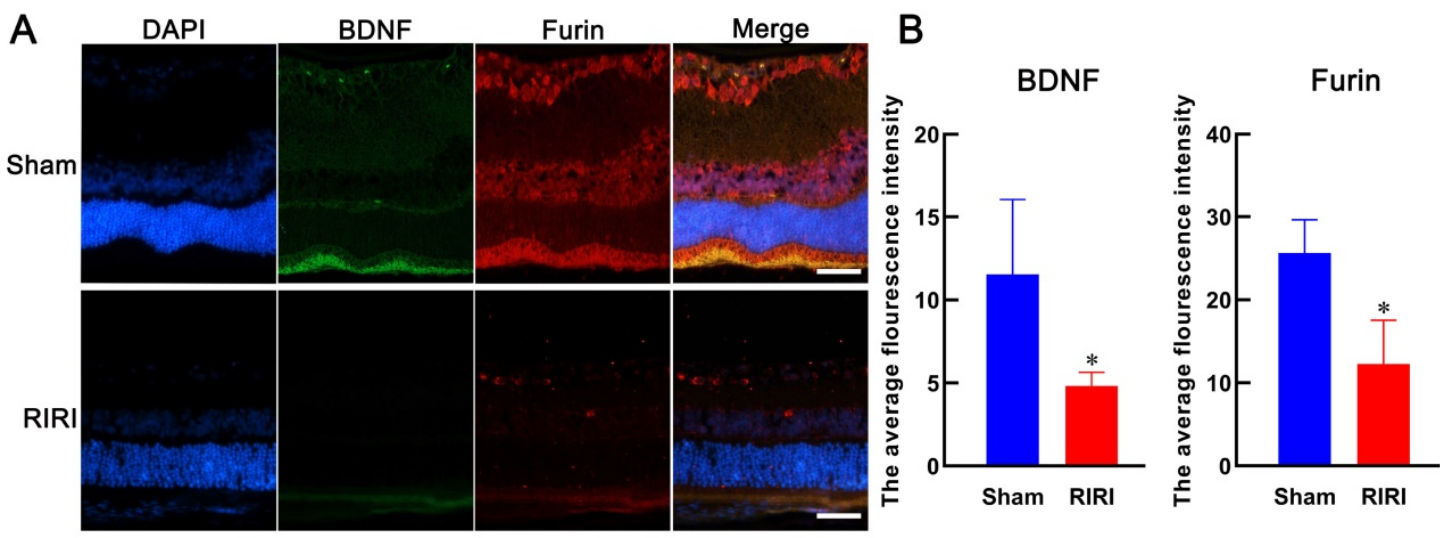

Figure 7. Expressions of Furin and BDNF in retinas of rat. Anti-BDNF (green stained) and anti-Furin (red stained) were used to detect their expression levels in retina by immunofluorescence. Both of them were deduced after RIRI. The average flourescence intensity was calculated by Image J and the histogram was made by GraphPad Prism 8 . *P $<0.05$ for RIRI compared with Sham by T-test $(n=3$ determinations for each). Bars $=50 \mu \mathrm{m}$. 
As a nerve growth factor, BDNF plays an important role in the growth and development of nerve cells and the maintenance of nerve cell survival. A series of studies have documented that the decrease of BDNF in protecting neuronal survival following diverse types of retinal injuries [7-8]. It is reasonable to believe that increasing BDNF levels can improve nerve cell survival based on not only restoring normal BDNF levels in retina tissue to maintain nerve cell vitality but also using more BDNF to stimulate nerve cell growth. Compared with BDNF injection or expression, the DHF (a mimetic flavonoid of BDNF) administration has its advantages in crossing the blood brain barrier when administered systemically and inducing TrkB response more quickly, enduringly, and robustly [20]. Despite in vitro studies suggest protective effects of DHF against excitotoxic and oxidative stress on RGC lines and high glucose induced apoptosis on retinal pigment epithelial (RPE) cell [21], few studies have explored the neuroprotective effects of DHF in the stressed retina. We have examined that DHF administration ameliorates apoptosis level of retina induced by RIRI. Similar to our findings, recent studies have shown that DHF protects immature retina against hypoxic-ischemic injury [12], protects retinal ganglion cells against chronic intermittent hypoxia [13] and intraorbital optic nerve transection [14], and inhibits optic nerve degeneration in Wolfram syndrome rat model [22].

The retina is a component of the central nervous system (CNS) and its stress response is like that of CNS. Pathological conditions such as ischemia, neurodegeneration and inflammation lead to activation of astrocytes are associated with an up-regulation of the intermediate filament GFAP [16-18]. Previous reports indicated that RIRI led to a higher expression level and immune mobility of GFAP $[23,24]$. We also found that astrocytes were activated by detecting GFAP immunofluorescence in RIRI retina and the GFAP mRNA was also up-regulated in RNA-seq analysis and qRT-PCR confirmation. Moreover, DHF can inhibit the expression of GFAP, indicating that DHF can reduce reactivation of astrocytes after RIRI. We found the NF-kB mediated inflammatory signaling pathway was activated in RIRI reversed by DHF treatment. Previous reports indicated that the activated astrocytes secreted inflammatory factors such as TNF- $\alpha$, IL-1 $\beta$, IL-6, IFN $\gamma$, and COX-2, which in turn further promoted astrocyte activation, forming a vicious cycle [25]. We speculated that DHF might inhibit the activity of astrocytes through Akt/NF-kB signaling and reduced the levels of inflammatory factors to protect the nerve cells in ischemic retina like previous report about the roles of RNase on RIRI [24]. However, the precise mechanism on DHF and reactivation of astrocytes still needs to be explored in further study.

Previous studies have shown that DHF has neuroprotective effects with increasing nerve cell survival neurotrophic activities in psychiatric diseases by mediating MAPK/Erk, PI3K/Akt, and PLCY signaling pathways following TrkB activation [26, 27]. DHF could have a similar effect in TrkB signaling which agrees with our present findings. Our data showed that DHF promoted TrkB phosphorylation and up-regulated the expression of BDNF, and increased the Erk phosphorylation and Akt phosphorylation level. However, DHF protected RIRI retina via not only TrkB signaling pathway but also other mechanisms such as antioxidant and antiinflammatory effects [28]. Moreover, our results showed that RIRI increased the BDNF precursor (proBDNF) level and decreased the mature BDNF (mBDNF) level, which increased neuronal cell apoptosis. ProBDNF can mediate the apoptosis of nerve cells after binding to the p75NTR receptor [29]. Interestingly, the proBDNF and mBDNF levels were restored to normal conditions after systematic DHF administration. It was speculated that the function of proprotein convertases like Furin that activates the precursor protein into a biologically active form was returned to normal in RIRI retina under systematic DHF administration [30,31]. Furin protein could convert proBDNF to mBDNF, which not only promoted the survival of nerve cells and the growth of axons and dendrites by activating mBNDF/TrkB pathway but also reduced nerve cell apoptosis by inhibiting proBDNF/P75NTR pathway. In some studies, it has been found that MMP activation can lead to increased BDNF release and TrkB activation in cortical neuron cultures [32,33]. The increase of intracellular zinc levels can increase MMP activity and BDNF expression [34]. In the future study, the relationship between BDNF and Furin (or MMP) needs to be clarified in the pathological process of RIRI, which could be helpful in delaying the apoptosis of optic nerve cells as much as possible and promoting the survival of retinal nerve cells, and provide more beneficial treatment method for protecting the visual function of RIRI patients.

In this study, RNA sequencing was used to discover differently expressed genes in RIRI and to further explore the genes and enriched pathways that were reversed by DHF administration. We found a lot of differently expressed genes have been discovered to be involved in RIRI. For example, Gfap and Casp3 were up-regulated after RIRI. KEGG analysis also showed that the NF-kappa B signaling pathway, 
Jak-STAT signaling pathway, Toll-like receptor signaling pathway, PI3K-Akt signaling pathway, TNF signaling pathway, MAPK signaling pathway, Calcium signaling pathway, cAMP signaling pathway, and Apoptosis were significantly enriched. These findings were in agreement with previous studies [12,35-41]. Different expression profile between RIRI and DHF+RIRI revealed reversed pathways responsible for DHF treatment. Several reversed pathways were overlapped with DHF induced BDNF/TrkB signaling, such as PI3K-Akt signaling pathway and Ras signaling pathway. And the other reversed pathways were indirect effects with the RIRI improvement and were also found in other studies, such as Toll-like receptor signaling pathway and NF-kappa B signaling pathway $[40,41]$.

In summary, the present study revealed that systemic administration of DHF activates the BDNF/TrkB/NF-kB pathway to alleviate apoptosis and inflammation of RIRI to the retina and ganglion cells. Moreover, the reactivation of astrocyte was inhibited and $\mathrm{mBDNF} /$ proBDNF ratio was increased after DHF administration which led to decreased levels of apoptosis and increased levels of nerve cell survival. Therefore, the DHF treatment could be used as a potential therapeutic drug for ischemic ophthalmopathy in clinical practice.

\section{Supplementary Material}

Supplementary figure S1 and table S3.

https://www.medsci.org/v19p0013s1.pdf

Supplementary table S1.

https://www.medsci.org/v19p0013s2.xlsx

Supplementary table S2.

https://www.medsci.org/v19p0013s3.xlsx

\section{Acknowledgments}

This work was supported by Health Commission of Hubei Province (ZY2021M043). We also thank Dr. Gang $\mathrm{Li}$ and Dr. Haoli Ma from Department of Biological Repositories, Zhongnan Hospital of Wuhan University for their technical assistance. We also thank Dr. Jiangtao $\mathrm{Yu}$ for his assistance in creating the TOC figure with BioRender.com.

\section{Author Contributions}

Yiqiao Xing conceived and designed this study and revised the manuscript; Aihua $\mathrm{Yu}$ and Shun Wang performed the most experiments and wrote manuscript; Aihua Yu, Shun Wang, and Mengyao Han analyzed the data; Mengyao Han and Kun Shao contributed reagents/materials/analysis tools.

\section{Competing Interests}

The authors have declared that no competing interest exists.

\section{References}

1. Nebbioso M, Franzone F, Greco A, et al. Recent Advances and Disputes About Curcumin in Retinal Diseases. Clin Ophthalmol. 2021; 15:2553-2571.

2. Minhas G, Sharma J, Khan N. Cellular Stress Response and Immune Signaling in Retinal Ischemia-Reperfusion Injury. Front Immunol. 2016; 7:444.

3. Barzegar M, Kaur G, Gavins FNE, et al. Potential therapeutic roles of stem cells in ischemia-reperfusion injury. Stem Cell Res. 2019; 37:101421.

4. Pinzón-Duarte G, Arango-González B, Guenther $\mathrm{E}$ et al. Effects of brain-derived neurotrophic factor on cell survival, differentiation and patterning of neuronal connections and Müller glia cells in the developing retina. Eur J Neurosci. 2004; 19(6):1475-1484.

5. Huang EJ, Reichardt LF. Trk receptors: roles in neuronal signal transduction. Annu Rev Biochem. 2003; 72:609-642

6. Liu X, Grishanin RN, Tolwani RJ, et al. Brain-derived neurotrophic factor and TrkB modulate visual experience-dependent refinement of neuronal pathways in retina. J Neurosci. 2007; 27(27):7256-7267.

7. Kurokawa T, Katai N, Shibuki H, et al. BDNF diminishes caspase-2 but not c-Jun immunoreactivity of neurons in retinal ganglion cell layer after transient ischemia. Invest Ophthalmol Vis Sci. 1999;40(12):3006-3011.

8. Di Polo A, Aigner LJ, Dunn RJ, et al. Prolonged delivery of brain-derived neurotrophic factor by adenovirus-infected Müller cells temporarily rescues injured retinal ganglion cells. Proc Natl Acad Sci U S A. 1998; 95(7):3978-3983.

9. Taylor AR, Gifondorwa DJ, Robinson MB, et al. Motoneuron programmed cell death in response to proBDNF. Dev Neurobiol. 2012; 72(5):699-712.

10. Koshimizu H, Kiyosue K, Hara T, et al. Multiple functions of precursor BDNF to CNS neurons: negative regulation of neurite growth, spine formation and cell survival. Mol Brain. 2009; 2:27.

11. Vidal-Sanz M, Galindo-Romero C, Valiente-Soriano FJ, et al. Shared and Differential Retinal Responses against Optic Nerve Injury and Ocular Hypertension. Front Neurosci. 2017; 11:235.

12. Huang HM, Huang CC, Tsai MH, et al. Systemic 7,8-Dihydroxyflavone Treatment Protects Immature Retinas Against Hypoxic-Ischemic Injury via Müller Glia Regeneration and MAPK/ERK Activation. Invest Ophthalmol Vis Sci. 2018; 59(7):3124-3135.

13. Fang YY, Luo M, Yue S, et al. 7,8-Dihydroxyflavone protects retinal ganglion cells against chronic intermittent hypoxia-induced oxidative stress damage via activation of the BDNF/TrkB signaling pathway. Sleep Breath. 2021; [Epub ahead of print].

14. Vidal-Villegas B, Di Pierdomenico J, Gallego-Ortega A, et al. Systemic treatment with 7,8-Dihydroxiflavone activates TtkB and affords protection of two different retinal ganglion cell populations against axotomy in adult rats. Exp Eye Res. 2021; 210:108694.

15. Liu R, Li G, Ma H, Zhou X, et al. Transcriptome profiling of the diaphragm in a controlled mechanical ventilation model reveals key genes involved in ventilator-induced diaphragmatic dysfunction. BMC Genomics. 2021; 22(1):472

16. Eddleston M, Mucke L. Molecular profile of reactive astrocytes--implications for their role in neurologic disease. Neuroscience. 1993; 54(1):15-36.

17. Wang X, Tay SS, Ng YK. An immunohistochemical study of neuronal and glial cell reactions in retinae of rats with experimental glaucoma. Exp Brain Res. 2000; 132(4):476-484

18. Wang L, Cioffi GA, Cull G, et al. Immunohistologic evidence for retinal glial cell changes in human glaucoma. Invest Ophthalmol Vis Sci. 2002; 43(4):1088-1094.

19. Levkovitch-Verbin H. Animal models of optic nerve diseases. Eye (Lond). 2004; 18(11):1066-1074.

20. Zhang Z, Liu X, Schroeder JP, et al. 7,8-dihydroxyflavone prevents synaptic loss and memory deficits in a mouse model of Alzheimer's disease. Neuropsychopharmacology. 2014; 39(3):638-650.

21. $\mathrm{Yu} X$, Liu $\mathrm{O}$, Wang $\mathrm{X}$, Liu $\mathrm{H}$, Wang $\mathrm{Y}, 7,8$-Dihydroxyflavone ameliorates high-glucose induced diabetic apoptosis in human retinal pigment epithelial cells by activating TrkB. Biochem Biophys Res Commun. 2018; 495(1):922-927.

22. Seppa K, Jagomäe $T$, Kukker KG, et al. Liraglutide, 7,8-DHF and their co-treatment prevents loss of vision and cognitive decline in a Wolfram syndrome rat model. Sci Rep. 2021; 11(1):2275.

23. $\mathrm{Li} N$, Wang $\mathrm{F}$, Zhang $\mathrm{Q}$ et al. Rapamycin mediates $m$ TOR signaling in reactive astrocytes and reduces retinal ganglion cell loss. Exp Eye Res. 2018; 176:10-19.

24. Han Y, Bing Zhu X, Ye Y, Yu Deng K, Yang Zhang X, Ping Song Y. Ribonuclease attenuates retinal ischemia reperfusion injury through inhibition of inflammatory response and apoptosis in mice. Int Immunopharmacol. 2020. $85: 106608$

25. Linnerbauer M, Wheeler MA, Quintana FJ. Astrocyte Crosstalk in CNS Inflammation. Neuron. 2020;108(4):608-622.

26. Liu $\mathrm{X}$, Chan $\mathrm{CB}$, Jang SW, et al. A synthetic 7,8-dihydroxyflavone derivative promotes neurogenesis and exhibits potent antidepressant effect. J Med Chem. 2010; 53(23):8274-8286 
27. García-Díaz Barriga G, Giralt A, Anglada-Huguet $M$, et al. 7,8-dihydroxyflavone ameliorates cognitive and motor deficits in a Huntington's disease mouse model through specific activation of the PLC $\gamma 1$ pathway. Hum Mol Genet. 2017; 26(16):3144-3160.

28. Liu C, Chan CB, Ye K. 7,8-dihydroxyflavone, a small molecular TrkB agonist, is useful for treating various BDNF-implicated human disorders. Transl Neurodegener. 2016; 5:2

29. Je HS, Yang F, Ji Y, et al. ProBDNF and mature BDNF as punishment and reward signals for synapse elimination at mouse neuromuscular junctions. J Neurosci. 2013; 33(24):9957-9962.

30. Dahms SO, Arciniega M, Steinmetzer T, Huber R, Than ME. Structure of the unliganded form of the proprotein convertase furin suggests activation by a substrate-induced mechanism. Proc Natl Acad Sci U S A. 2016; 113(40):11196-11201.

31. Henrich S, Cameron A, Bourenkov GP, et al. The crystal structure of the proprotein processing proteinase furin explains its stringent specificity. Nat Struct Biol. 2003;10(7):520-526.

32. Susan-Resiga D, Essalmani R, Hamelin J, et al. Furin is the major processing enzyme of the cardiac-specific growth factor bone morphogenetic protein 10 . J Biol Chem. 2011; 286(26):22785-22794.

33. Chen $Y$, Zhang J, Deng M. Furin mediates brain-derived neurotrophic factor upregulation in cultured rat astrocytes exposed to oxygen-glucose deprivation. J Neurosci Res. 2015; 93(1):189-194.

34. Gupta VK, You Y, Gupta VB, et al. TrkB receptor signalling: implications in neurodegenerative, psychiatric and proliferative disorders. Int J Mol Sci. 2013; 14(5):10122-10142.

35. Kadzielawa K, Mathew B, Stelman CR, et al. Gene expression in retinal ischemic post-conditioning. Graefes Arch Clin Exp Ophthalmol. 2018; 256(5):935-949.

36. Wu J, Wang R, Yang D, et al. Hydrogen postconditioning promotes survival of rat retinal ganglion cells against ischemia/reperfusion injury through the PI3K/Akt pathway. Biochem Biophys Res Commun. 2018; 495(4):2462-2468.

37. Du HY, Wang R, Li JL, et al. Ligustrazine induces viability, suppresses apoptosis and autophagy of retinal ganglion cells with ischemia/reperfusion injury through the PI3K/Akt/mTOR signaling pathway. Bioengineered. 2021; 12(1):507-515.

38. Husain S, Liou GI, Crosson CE. Opioid receptor activation: suppression of ischemia/reperfusion-induced production of TNF-a in the retina. Invest Ophthalmol Vis Sci. 2011; 52(5):2577-2583.

39. Liu L, Jiang Y, Steinle JJ. Epac1 protects the retina against ischemia/reperfusion-induced neuronal and vascular damage. PLoS One. 2018;13(9):e0204346. Published 2018 Sep 20. doi:10.1371/journal.pone.0204346

40. Guan L, Li C, Zhang Y, et al. Puerarin ameliorates retinal ganglion cell damage induced by retinal ischemia/reperfusion through inhibiting the activation of TLR4/NLRP3 inflammasome. Life Sci. 2020; 256:117935.

41. Lin $\mathrm{C}, \mathrm{Wu} \mathrm{F}$, Zheng $\mathrm{T}$, et al. Kaempferol attenuates retinal ganglion cell death by suppressing NLRP1/NLRP3 inflammasomes and caspase-8 via JNK and NF-kB pathways in acute glaucoma. Eye (Lond). 2019; 33(5):777-784. 